\title{
Effect of Velocity Anisotropy in Shale on the Acoustic Emission Events Matching and Location
}

\author{
Peng Wang, Feng Zhang * and Xiang-Yang Li
}

China University of Petroleum, Beijing, China

\section{OPEN ACCESS}

Edited by:

Jidong Yang,

China University of Petroleum

Huadong, China

Reviewed by:

Song JIn,

China University of Geosciences

Wuhan, China

Yaojun Wang,

University of Electronic Science and

Technology of China, China

Zhiqi Guo,

Jilin University, China

*Correspondence:

Feng Zhang

zhangfeng@cup.edu.cn

Specialty section:

This article was submitted to

Solid Earth Geophysics,

a section of the journal

Frontiers in Earth Science

Received: 07 November 2021

Accepted: 20 December 2021

Published: 11 February 2022

Citation:

Wang $P$, Zhang $F$ and

Li X-Y (2022) Effect of Velocity

Anisotropy in Shale on the Acoustic

Emission Events Matching

and Location.

Front. Earth Sci. 9:810578.

doi: 10.3389/feart.2021.810578
Accurate source event location is important in fracturing monitoring and characterization. Velocity anisotropy has a great influence on both events matching and events location. Failure to take into account the velocity anisotropy can lead to huge errors in locating events. In this article, we have presented an experimental study on lower Silurian shale from the Sichuan Basin. The experimental observations include ultrasonic measurements, acoustic emissions (AEs) in a three-point bend experiment, and CT scanning of the original sample and the fractured sample. The ultrasonic measurements show that the shale sample has strong velocity anisotropy. Initially, AEs are analyzed using the conventional event-matching method and event location method (Geiger's method), and the detected events are compared to the $X$-ray image of the fracture. Event-matching aims to obtain $A E$ signals from the same source event and thus assists in selecting valid $A E$ signals that come from the same source and are received by at least four sensors, to determine the location of the source. Although many reliable signals are obtained by isotropic event-matching, fewer sources were located than expected, and the event location results did not match the fracture distribution. To address this problem, an improved event-matching method is proposed using a stricter matching threshold based on directional velocity rather than a single threshold same for all directions. In addition, we propose an improved Geiger's method using the anisotropic velocity model. The new methods located more sources that better match fracture distribution than the results of the isotropic method. We have concluded that both event-matching and the source location of the fracturing are largely influenced by velocity anisotropy, and thus in practice, the velocity anisotropy information obtained from various measurements (e.g., laboratory measurements, well logs, VSP, and velocity analysis of reflected seismic surveys) should be involved in both processing procedures. This study can be useful to provide some background for monitoring and predicting dynamic geo-hazards in relation to the AE method.

Keywords: acoustic emission, microseismic, shale, anisotropy, event-matching, source location

\section{INTRODUCTION}

Seismic source location is important in earthquake research, fracturing monitoring, and acoustic emission (AE) experiment. Triggering events can be located by minimizing an objective function in terms of the difference between observed and theoretical arrival times (Geiger, 1912; Ge, 2013; Wuestefeld et al., 2018). The reliable location of an event depends on an accurate velocity model. 


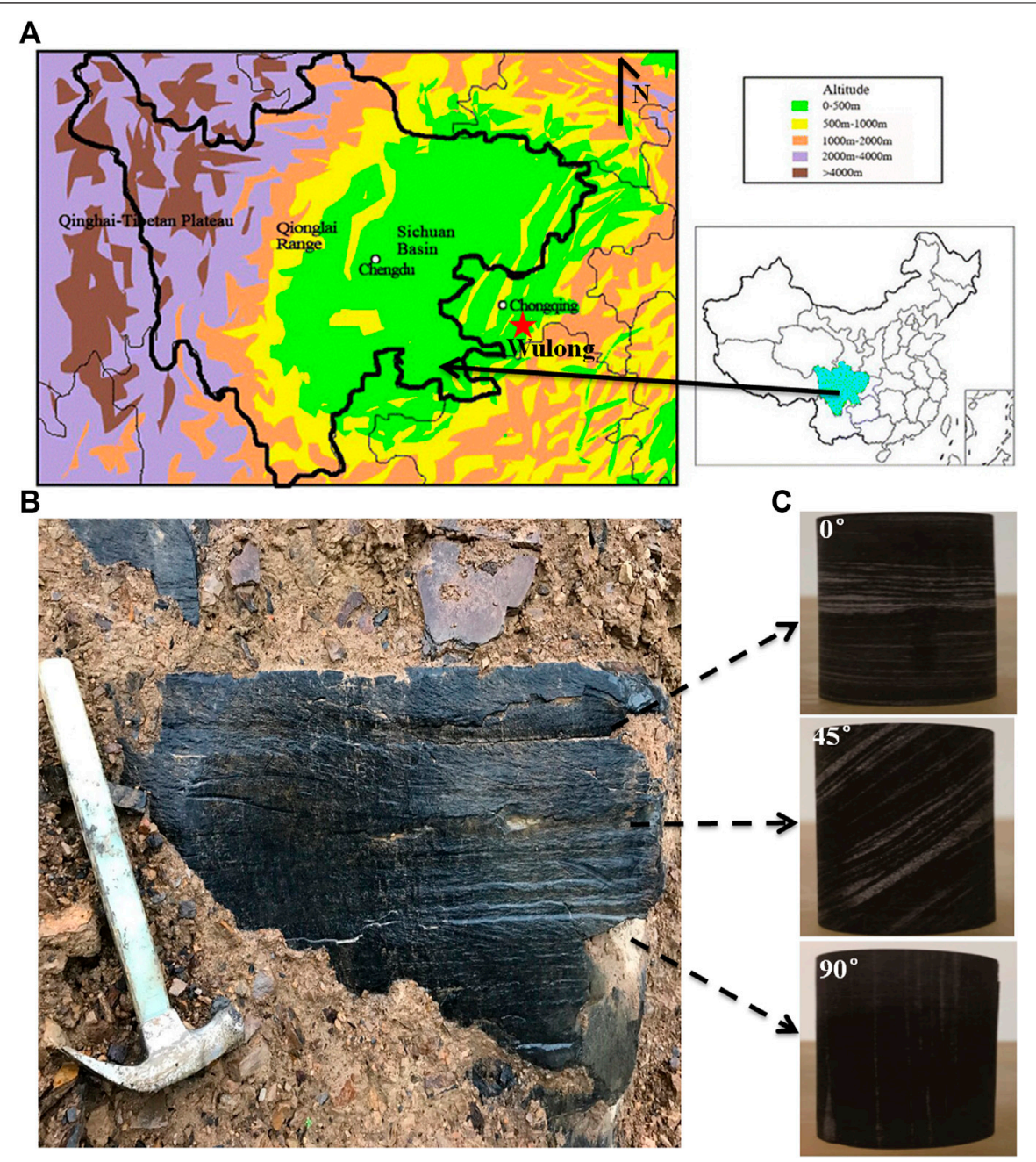

FIGURE 1 | (A) Shale samples collected from Wulong, southeast of Sichuan Basin. (B) Silurian Longmaxi shale outcrops. (C) Cores are cut in three different directions.

TABLE 1 | Mineral composition of the Silurian Longmaxi shale sample (measured using an X-ray fluorescence spectrometer).

\begin{tabular}{|c|c|c|c|c|c|c|c|}
\hline Mineral & Quartz & K-feldspar & Plagioclase & Calcite & Dolomite & Pyrite & Clay \\
\hline Fraction (\%) & 58.6 & 1.9 & 5.5 & 2.3 & 6.9 & 3.9 & 20.9 \\
\hline
\end{tabular}

TABLE 2 | Velocities of P- and S-waves measured in three directions.

\begin{tabular}{lccc} 
Angle to bedding $\left.\mathbf{(}^{\circ}\right)$ & $\mathbf{0}$ & $\mathbf{4 5}$ & $\mathbf{9 0}$ \\
\hline $\mathrm{Vp}(\mathrm{km} / \mathrm{s})$ & 3.54 & 3.96 & 4.51 \\
$\mathrm{Vs}(\mathrm{km} / \mathrm{s})$ & 2.24 & 2.74 & 2.60 \\
$\rho\left(\mathrm{g} / \mathrm{cm}^{3}\right)$ & 2.51 & 2.52 & 2.53 \\
\hline
\end{tabular}

Current source location methods assume homogenous and isotropic velocity models (King and Talebi, 2007; Zhou et al., 2017). However, shale is observed to have strong anisotropy caused by preferentially orientated clay platelets and other integrated factors on a small scale (Vernik and Nur, 1992;
Lonardelli et al., 2007; Zhang, 2017; Zhang et al., 2017). Anisotropic shale can be modeled as a VTI (vertical transverse isotropy) medium in which the velocity of the acoustic wave perpendicular to the shale bedding is less than the velocity parallel to the shale bedding. The magnitude of shale anisotropy can be up to $40 \%$, and thus the effect of anisotropy must be taken into account in source location and event-matching.

Event-matching obtaining valid $\mathrm{AE}$ signals is a necessary procedure to locate the source. Valid AE signals mean those signals are from the same source and are received by at least four receivers because four unknown source parameters including the location coordinates $\left(x_{0}, y_{0}, z_{0}\right)$ and the origin time $\left(t_{0}\right)$ need to be determined. Accurate events matching can be difficult since 


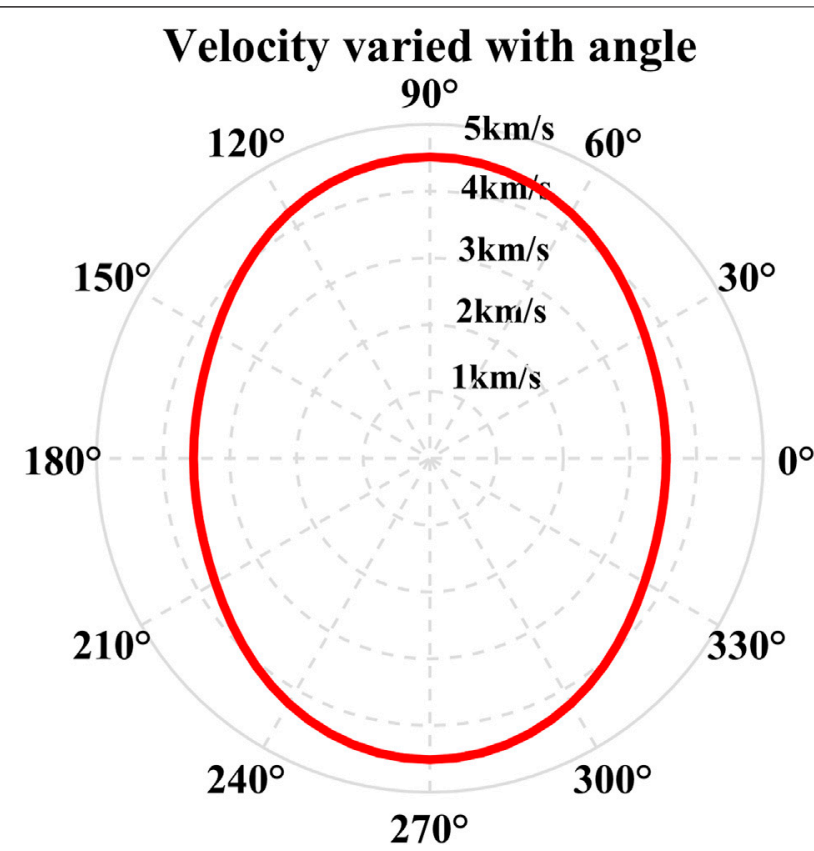

FIGURE 2 | Variation of velocity with an angle to the axis of symmetry.

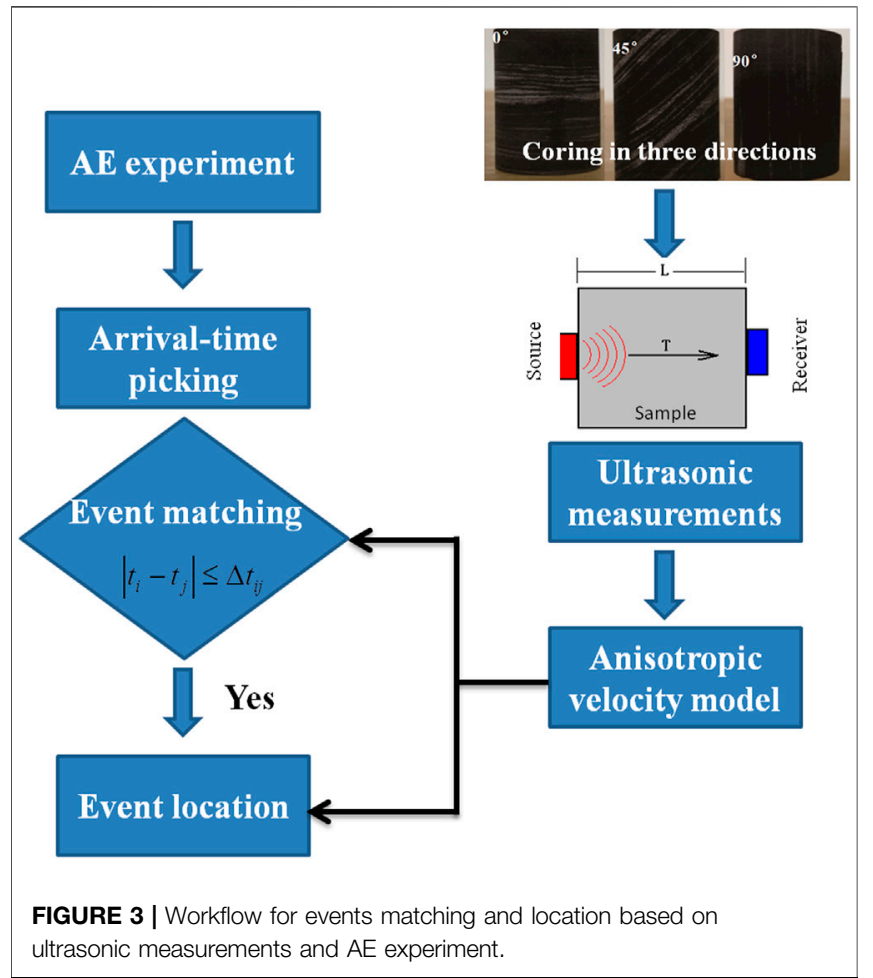

measurements, cross-well measurement using threecomponent sensors, and advanced dipole sonic, which is mainly applicable to field microseismic data and makes it difficult to obtain anisotropic parameters in $\mathrm{AE}$ experiments in the laboratory; therefore, we use a core measurement method to obtain an anisotropic velocity model. An improved Geiger's method is proposed that corrects the anisotropic velocity instead of using a constant velocity during each iteration.

In this article, we first perform ultrasonic measurements on a shale sample from the lower Silurian shale formation in the southern Sichuan Basin to investigate its elastic properties. An acoustic emission experiment is then carried out on a shale sample. The number of located sources using traditional event-matching and Geiger's method is incompatible with the $\mathrm{X}$-ray image of the fracture. To address this issue, we study the effect of anisotropy on event-matching and propose an improved event-matching method based on a triangulation method considering velocity anisotropy. The newly proposed matching method greatly improves data-processing efficiency by reducing invalid redundant AE events. Finally, we propose an improved Geiger's method by taking into account velocity anisotropy and verify the accuracy of the location results based on a CT scan.

\section{ULTRASONIC MEASUREMENTS OF A SHALE SAMPLE}

Shale samples are collected from an outcrop of the Longmaxi formation in Wulong County, Chongqing City (Figure 1A,B). 


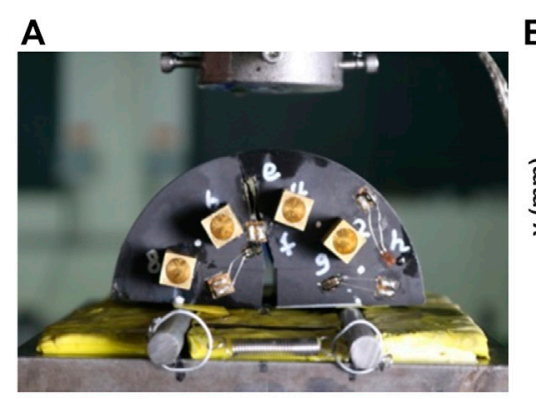

D

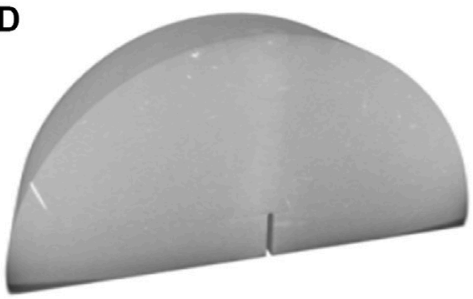

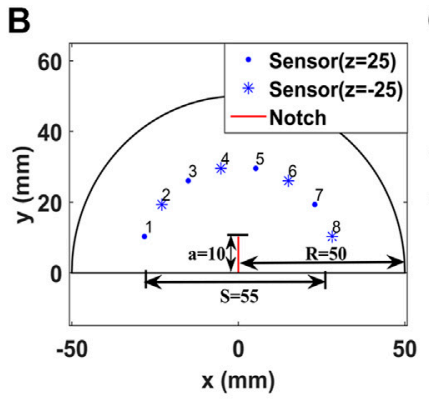
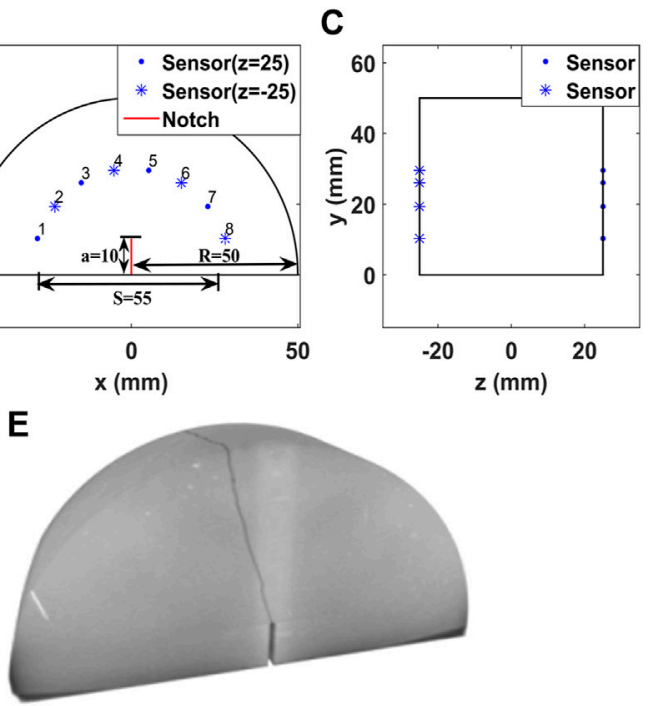

FIGURE 4 | (A) Notched semicircular bend (NSCB) shale sample after unaxial loading. (B) The front view of sample geometry and eight AE sensors. The distance between the two supporting(S) is $55 \mathrm{~mm}$ and a $10 \mathrm{~mm}$ notch is made in the middle of the lower part of the sample to cause a directed rupture. (C) The side view of sample geometry and four sensors are glued on the $z=25 \mathrm{~mm}$ plane (blue point) and the rest are on the $\mathrm{z}=-2 \mathrm{~mm}$ plane(blue asterisk).X-ray images of $C T$ scanning (D) and (E) after fracturing.

TABLE 3 | Basic parameters of the AE experiment.

\begin{tabular}{lc}
\hline Design parameter & Value \\
\hline Sample frequency & $5,000 \mathrm{kHz}$ \\
Sample point & 4,000 \\
Pre-sampling time & $150 \mathrm{\mu s}$ \\
Waveform threshold & $35 \mathrm{~dB}$ \\
Preamplifiers & $40 \mathrm{~dB}$ \\
Filter & $100 \mathrm{kHz}-400 \mathrm{kHz}$ \\
Loading rate & $0.01 \mathrm{~mm} / \mathrm{min}$
\end{tabular}

The density and porosity of the shale sample are measured as $2.52 \mathrm{~g} / \mathrm{cm}^{3}$ and $3.83 \%$, respectively. The mineral grades are shown in Table 1. Although the shale contains a large volume of quartz, the alignment of the clay mineral is considered to be the determining factor in causing the anisotropy of seismic velocities (Liu et al., 2019; Zhang, 2019). We used the method proposed by Vernik and Nur (1992) to measure P- and S-wave velocities for three cylindrical plugs cut in three directions (normal to bedding, $45^{\circ}$ to bedding, and parallel to bedding) from the sample (Figure 1C).

An ultrasonic pulse testing system is used to measure the velocities of $\mathrm{P}$ - and $\mathrm{S}$-waves (SH-wave) in a shale sample. The measurements were carried out at room temperature and pressure, the main frequencies of $\mathrm{P}$ - and S-wave transducers were 1 and $0.5 \mathrm{MHz}$, respectively, and the test error was less than $1 \%$. The measured velocities are shown in Table 2. The quantities $V_{P}\left(0^{\circ}\right)$ and $V_{S H}\left(0^{\circ}\right)$ represent the velocities of the $\mathrm{P}$ - and $\mathrm{SH}$ waves along the normal to the bedding, respectively. The quantities $V_{P}\left(45^{\circ}\right)$ and $V_{S H}\left(45^{\circ}\right)$ represent the velocities of $\mathrm{P}$ - and S-waves at an angle of $45^{\circ}$ to the bedding, respectively. The quantities $V_{P}\left(90^{\circ}\right)$ and $V_{S H}\left(90^{\circ}\right)$ represent the velocities of the $\mathrm{P}$ - and S-waves parallel to the bedding, respectively. It is seen that

$$
V_{P}\left(0^{\circ}\right)<V_{P}\left(45^{\circ}\right)<V_{P}\left(90^{\circ}\right)
$$

and

$V_{S H}\left(0^{\circ}\right)<V_{S H}\left(45^{\circ}\right)<V_{S H}\left(90^{\circ}\right)$. Thus, a shale sample has an equivalent VTI property and five independent elastic stiffness coefficients $C_{i j}$, which can be calculated using the measured velocity and density $\rho$ as (Wang, 2002; Mavko et al.,2003)

$\left\{\begin{array}{l}c_{11}=\rho V_{P}^{2}\left(90^{\circ}\right), \quad c_{12}=c_{11}-2 \rho V_{S H}{ }^{2}\left(90^{\circ}\right), \\ c_{33}=\rho V_{P}^{2}\left(0^{\circ}\right), \quad c_{44}=\rho V_{S H}{ }^{2}\left(0^{\circ}\right), \quad c_{66}=\frac{1}{2}\left(c_{11}-c_{12}\right), \\ c_{13}=-c_{44}+\sqrt{4 \rho^{2} V_{P}^{4}\left(45^{\circ}\right)+\left(c_{11}+c_{44}\right)\left(c_{33}+c_{44}\right)-2 \rho V_{P}{ }^{2}\left(45^{\circ}\right)\left(c_{11}+c_{33}+2 c_{44}\right)} .\end{array}\right.$

The P-wave velocity as a function of angle is calculated using the following equation:

$$
V_{P}(\alpha)=\left(c_{11} \sin ^{2} \alpha+c_{33} \cos ^{2} \alpha+c_{44}+\sqrt{M}\right)^{1 / 2}(2 \rho)^{-1 / 2},
$$

where $\alpha$ is the phase angle to the bedding normal and $M=\left[\left(c_{11}-c_{44}\right) \sin ^{2} \alpha-\left(c_{33}-c_{44}\right) \cos ^{2} \alpha\right]^{2}+\left(c_{13}+c_{44}\right)^{2} \sin ^{2} 2 \alpha$. The velocity of $\mathrm{P}$-wave, which varies depending on the direction, is shown in Figure 2 and is used for further event-matching and location using data from the $\mathrm{AE}$ experiment (Figure 3). Since in subsequent AE experiments the sensors have only one component, only the P-wave velocity is used to locate events. The kinematics of $\mathrm{P}$-wave in the TI medium weakly depends on the S-wave phase velocity (Alkhalifah, 1998; Jin and Stovas, 2018; Jin and Stovas, 2020). The magnitude of shale anisotropy can be represented by the $\mathrm{P}$-wave velocity anisotropy parameter $\varepsilon=$ $\left(C_{11}-C_{33}\right) / 2 C_{33}$ (Thomsen, 1986). The measured shale sample has strong $\mathrm{P}$-wave anisotropy, since its $\varepsilon$ is 0.27 . The densities of the three samples are almost the same, and slight deviations can be caused by heterogeneity. 

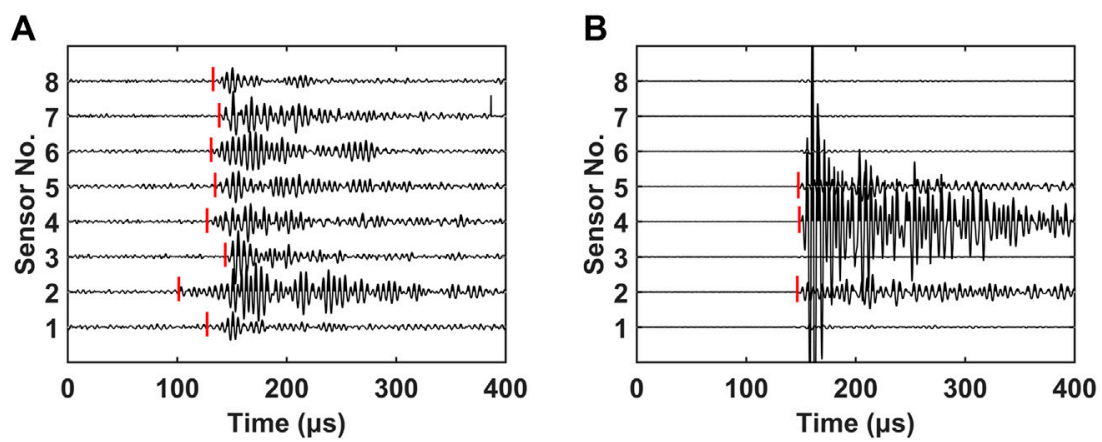

FIGURE 5 | (A) AE event recorded by eight sensors. The red line represents the selected arrival times. (B) AE event received by only three sensors (Sensor 2, 4, and 5).

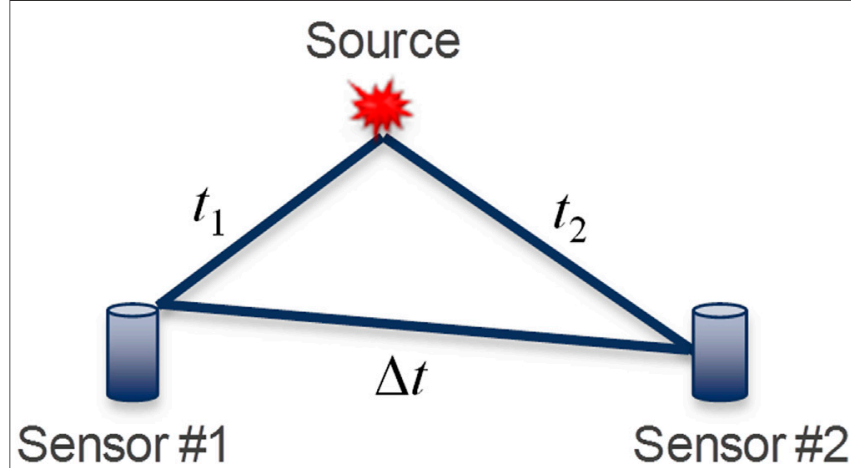

FIGURE 6 | Principle of the triangulation method of event-matching.

\section{ACOUSTIC EMISSION EXPERIMENTS}

A three-point bend acoustic emission experiment is carried out on a semicylindrical shale sample that is $50 \mathrm{~mm}$ thick and has a radius of $50 \mathrm{~mm}$ (Figures $\mathbf{4 A}, \mathbf{B}$ ). The sample before fracturing did not contain natural fractures. Its axial direction is perpendicular to the bedding. Compressive loads are applied to three points with a ratio of $0.01 \mathrm{~mm} / \mathrm{min}$ for 1 hour until the destruction of the sample. The AE signals are received by eight resonance-type sensors (SR150S) placed on the surface of the sample (Figure 4C). The sensors have a frequency range of $70 \sim 280 \mathrm{kHz}$ and a resonance frequency of $150 \mathrm{kHz}$. The main parameters of the AE experiment are shown in Table 3. The AE signal sampling frequency is $5,000 \mathrm{kHz}$ with a sampling interval of $0.2 \mu \mathrm{s}$, a waveform threshold of $35 \mathrm{~dB}$, a pre-sampling time of $150 \mu \mathrm{s}$, a sampling length of 4,000 points, and a total recording length of $800 \mu s$ for each acquisition segment. The eight $\mathrm{AE}$ sensors and $40-\mathrm{dB}$ preamplifiers were used in the tests, and $\mathrm{AE}$ signals exceeding $40 \mathrm{~dB}$ were captured during fracturing. CT scans are performed before and after rock breakdown to determine fracture distribution. The results of the CT X-ray images are shown in Figures 4D,E. These results will be used as the true fracture distribution for comparing source locations.

\section{EVENT-MATCHING FOR ANISOTROPIC MEDIA}

Event-matching aims to obtain AE signals from the same source event. It is carried out after the acquisition of the first arrivals (Figure 3). Ideally, an $\mathrm{AE}$ event is received and recorded by all eight sensors (Figure 5A). However, many $\mathrm{AE}$ events are received and recorded by only a few sensors (Figure 5B). This may be because these events are not strong enough to trigger all sensors for recording. To determine the location of the AE source, it is necessary to solve four unknown parameters, including the coordinates of the location $\left(x_{0}, y_{0}, z_{0}\right)$ and the origin time $\left(t_{0}\right)$. Therefore, valid AE signals are signals from the same source received by at least four sensors. Hence, reliable AE event-matching is crucial to locate the source.

Event-matching can be achieved using a triangulation rule as shown in Figure 6. The difference between the AE arrival times from the two sensors is compared with the time threshold $\Delta t$ to confirm if they are valid. The time threshold is usually constant and is expressed as the difference in travel time between the two farthest points or sensors in the sample (Feng et al., 2019). The arrival times of AE signals received by two sensors $\left(t_{1}\right.$ and $\left.t_{2}\right)$ correspond to two sides of the triangle. Knowing the coordinates of two sensors, it is possible to calculate the travel time from one sensor to the other as a "third side" using a known velocity model. The signals can be identified as from the same source event, if the following matching condition is met:

$$
\left|t_{1}-t_{2}\right| \leq \Delta t
$$

In this three-point bending experiment, assuming the sample is isotropic, $\Delta t$ is calculated as $30 \mu \mathrm{s}$ using a constant velocity $3.54 \mathrm{~km} / \mathrm{s}$. As shown in Figure 7A, signals are received by four sensors, but the differences in arrival times between them are much greater than $\Delta t$, so this set of signals is recognized as invalid based on the formula Eq. 3. In this article, the Akaike information criterion (AIC) (Maeda, 1985) is used to determine the time of the first 

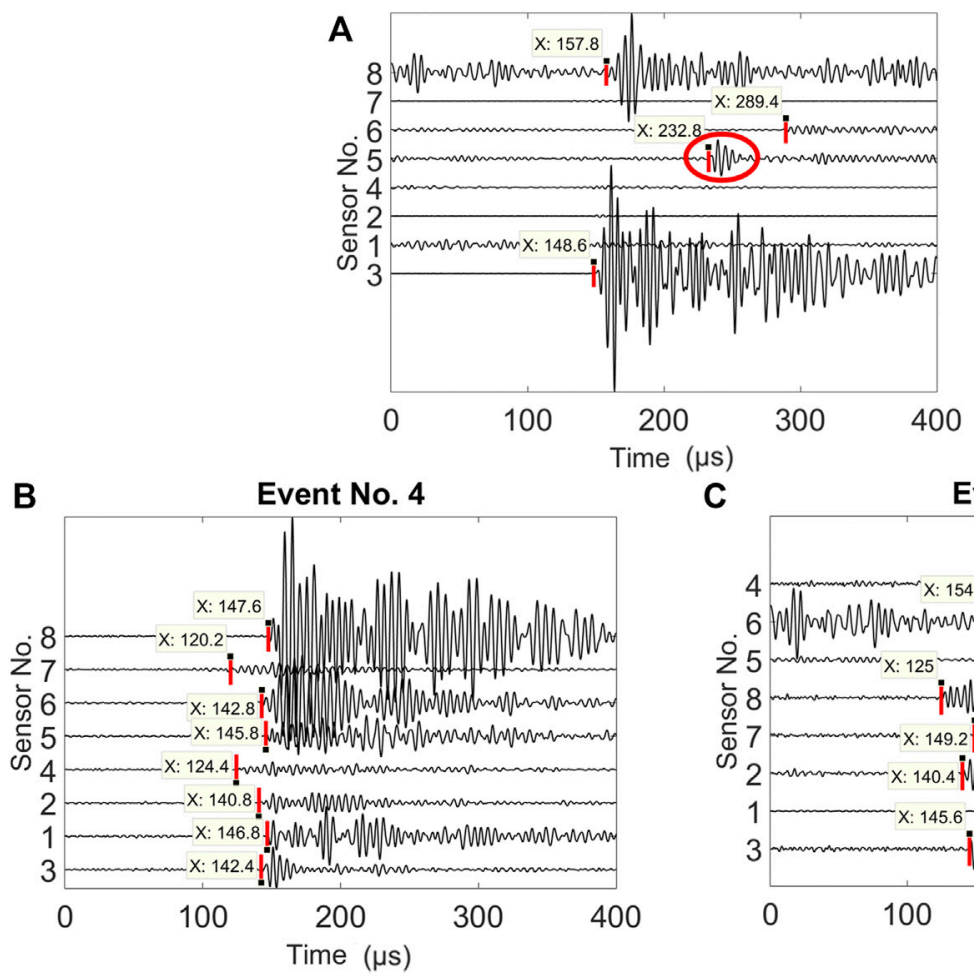

C

Event No. 12

D

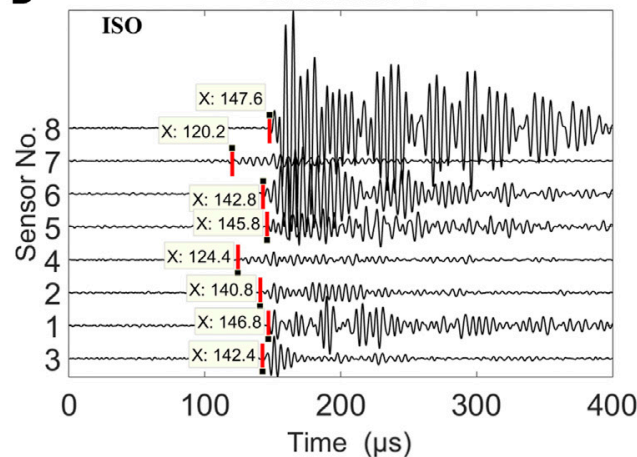

$\mathbf{F}$

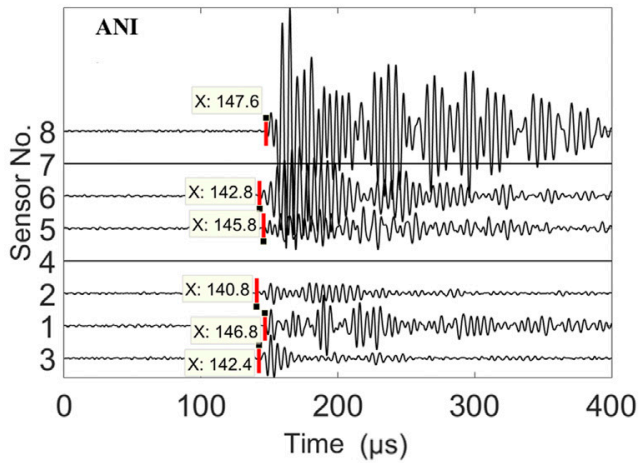

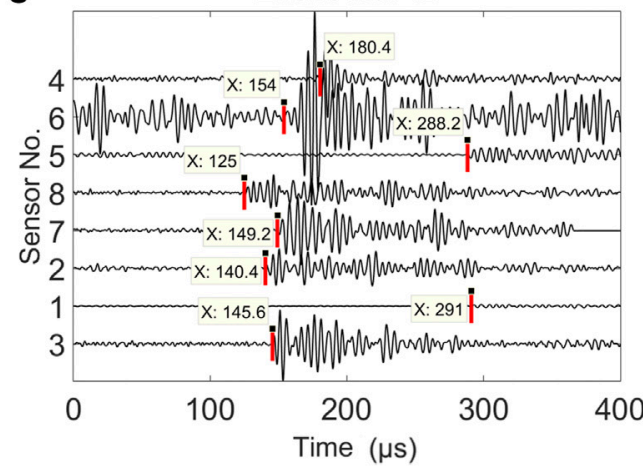

Event No. 12

E

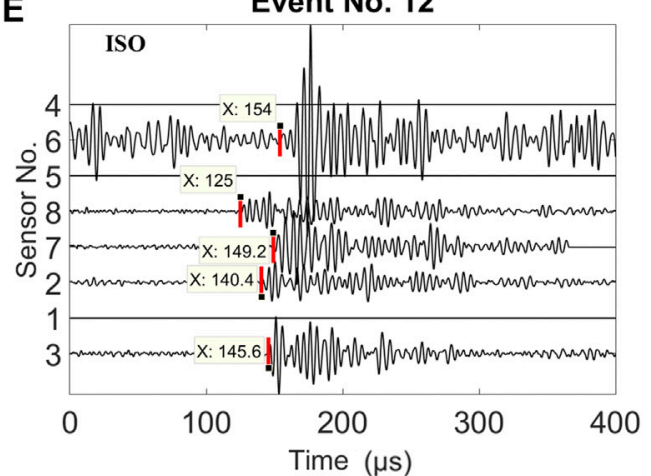

G

Event No. 12

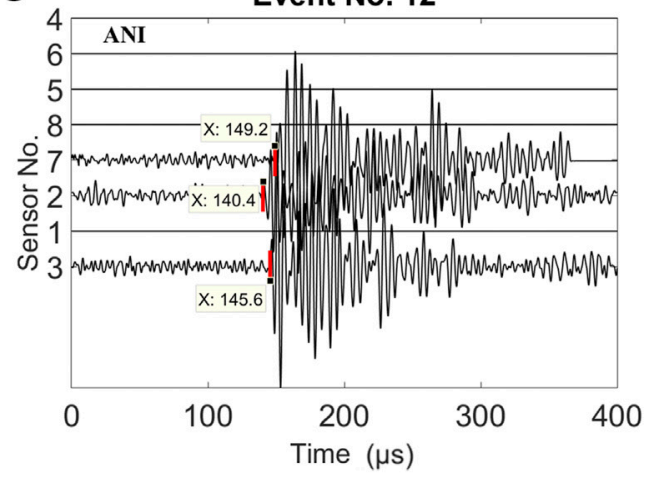

FIGURE 7 | (A) Signals received by four sensors are considered invalid. Red lines represent first arrivals, and the red ellipse in the sensor 5 represents the boundary reflection. (B) Event No. 4 and (C) Event No. 12 received by all eight sensors before event-matching. (D) Event No. 4 and (E) Event No. 12 after event-matching using the constant $\Delta t$ as $30 \mu \mathrm{s}$. (F) Event No. 4 and (G) Event No. 12 after event-matching using $\Delta t$ calculated using anisotropic velocity. 

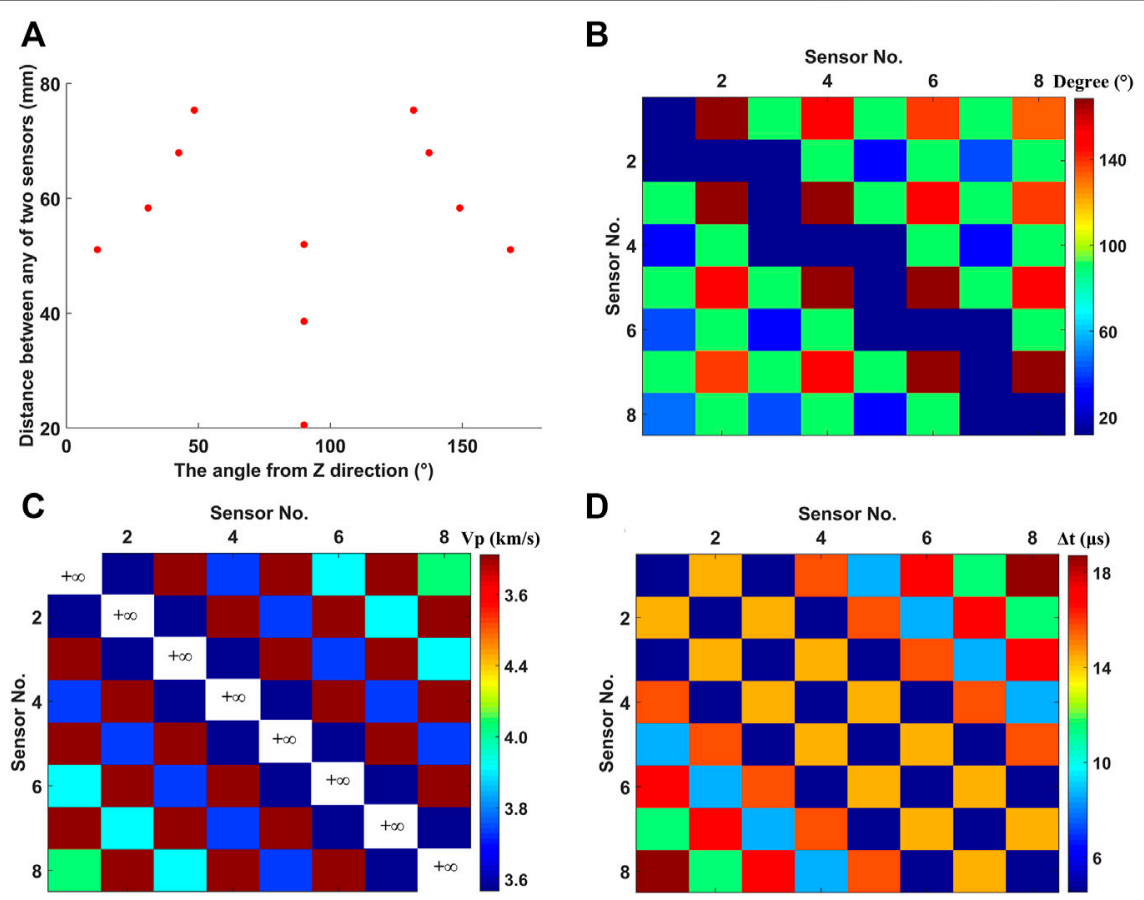

FIGURE 8 | (A) Relationship between the distances between two sensors and the angle from the Z direction in a three-point bend experiment. (B) Directions between either of the two sensors. (C) Velocity variation between either of the two sensors. (D) Differences in travel time between either of the two sensors. It is noted that the time differences $\left(\Delta t_{i i}\right)$ between the same sensors are equal to 0 , and the propagation velocity is infinite.
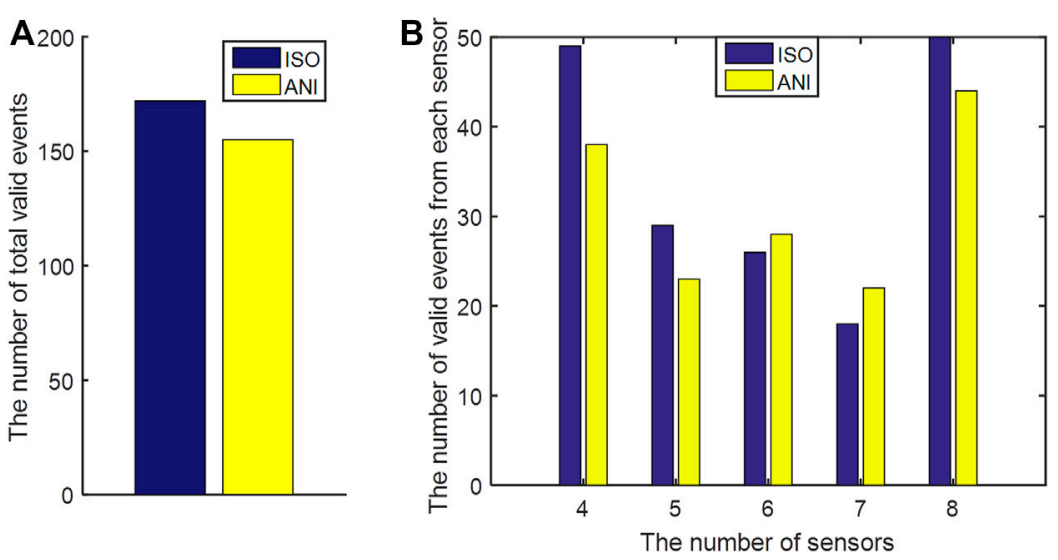

FIGURE 9 | (A) Total number of valid events matched using isotropic (ISO) and anisotropic (ANI) methods. (B) Number of valid events from each sensor, matched using ISO and ANI event-matching methods.

arrival of AE events. Although this method is convenient and highly effective, it can match redundant signals because received signals are mixed with boundary reflections or signals from other sources. As shown in Figures $7 \mathbf{B}, \mathbf{C}$, events No.4 and No.12 contain signals received by all eight sensors. If $\Delta t$ is used as $30 \mu$ s, eight signals and six signals can be matched for events No.4 and No. 12, respectively, and thus both events are considered valid.
However, neither of these two events can complete the subsequent location. Either the location result is outside the sample, or an unreliable solution is obtained. This is because redundant invalid events are matched as valid using a constant $\Delta t$, ignoring directional velocity variation.

To solve this problem, an improved matching condition is proposed, including the impact of anisotropy. In this $\mathrm{AE}$ experiment, sensors are arranged in a semicircle, and their 

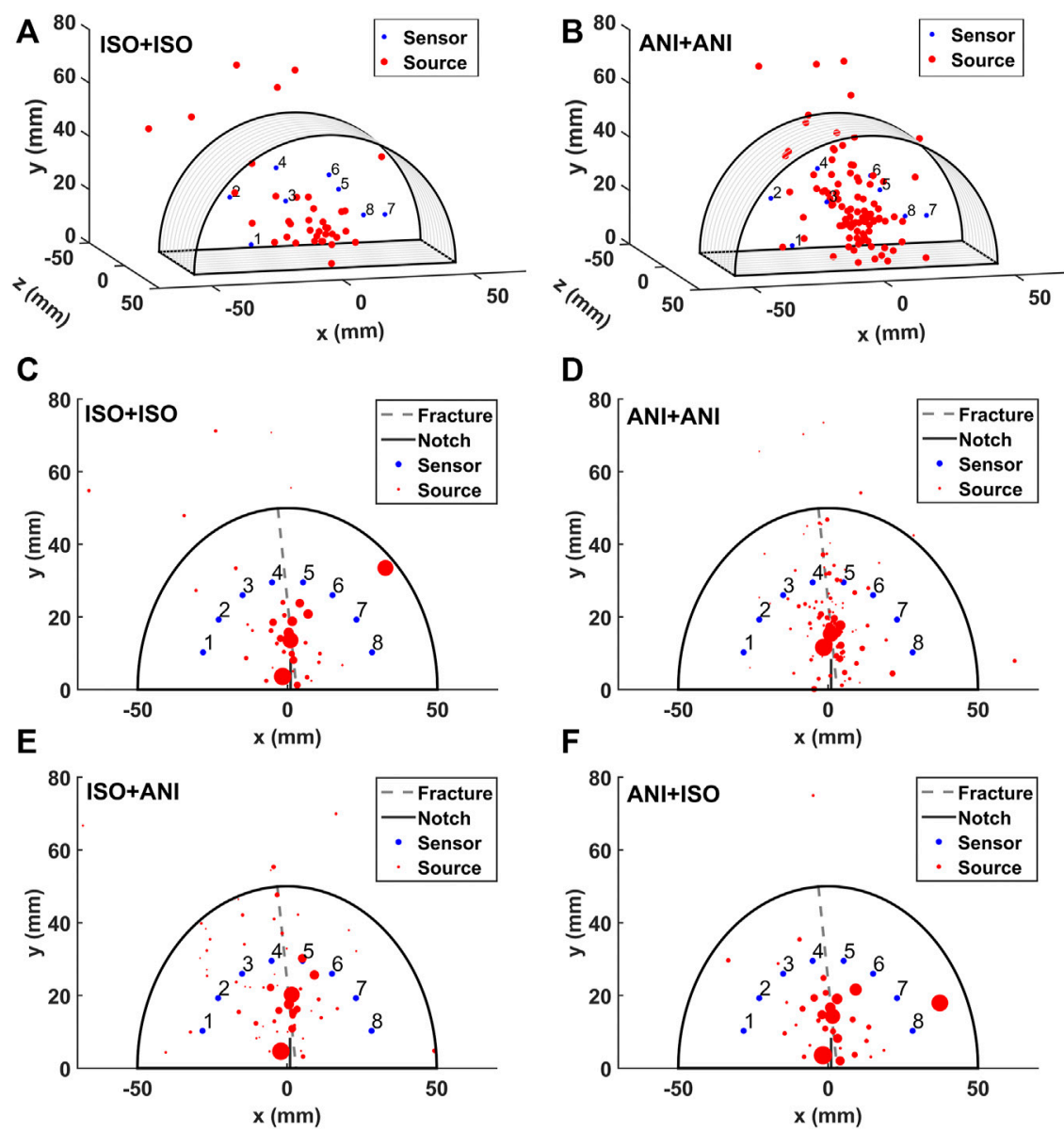

FIGURE 10 | Source location results (3D display) using (A) an isotropic matching and isotropic location (ISO+ISO) method and (B) an anisotropic matching and anisotropic location (ANI+ANI) method. Comparison of fracture and location results (Front view) using (C) isotropic matching and isotropic location (ISO + ISO), (D) anisotropic matching and anisotropic location (ANI + ANI), (E) isotropic matching and anisotropic location (ISO + ANI), and (F) anisotropic matching and isotropic location $(\mathrm{ANI}+\mathrm{ISO})$. The gray dashed lines represent the fracture, and the magnitude of source events is displayed using dots of various sizes.

angles are in a wide range (Figure 8A). As discussed in Eq. 2, the velocity of propagation of signals $V_{p}(\alpha)$ depends on the phase angle. Directional sines and cosines of the $i$ th sensor relative to the $j$ th sensor are respectively equal,

$\sin \alpha=\sqrt{\left(x_{i}-x_{j}\right)^{2}+\left(y_{i}-y_{j}\right)^{2}} / R_{i j}$ and $\cos \alpha=\left(z_{i}-z_{j}\right) / R_{i j}$,

where $\left(x_{i}, y_{i}, z_{i}\right)$ and $\left(x_{j}, y_{j}, z_{j}\right)$ are the coordinates of the $i$ th and the $j$ th sensor, respectively, and $R_{i j}$ represents the linear distance between any of the two sensors. Figure $\mathbf{8 B}$ shows the calculated value of $\alpha$ of either of two sensors using Eq. 4. Figure 8C shows calculated $V_{P}(\alpha)$ using the formula Eq. 2 based on ultrasonic measurements. The time threshold $\Delta t$ is now updated as

$$
\Delta t_{i j}=R_{i j} / V_{p}(\alpha),
$$

and the new matching condition is

$$
\left|t_{i}-t_{j}\right| \leq \Delta t_{i j}
$$

The calculated value of $\Delta t_{i j}$ between any of two sensors is shown in Figure 8D. The time differences between the two sensors range from 0 to $20 \mu \mathrm{s}$, which are less than the isotropic $\Delta t(30 \mu \mathrm{s})$.

Compared to the initial results, using the improved matching condition that takes into account the impact of anisotropy, fewer signals are matched (Figures 7F,G). Six signals are matched for event No. 4, which is still recognized as a valid $\mathrm{AE}$ event, while only three signals are matched for event No. 12 and thus cannot be used for locating since the number of signals is less than four. In general, the results of anisotropic matching remove redundant events and thus reduce the total number of effective events (Figure 9A). A total of 179 valid events were obtained using event-matching without taking into account the anisotropy effect, and a total of 153 valid events are obtained using the new event-matching method taking into account velocity anisotropy. In particular, the number of events received by the four sensors is significantly reduced (Figure 9B). The reason is because that the 

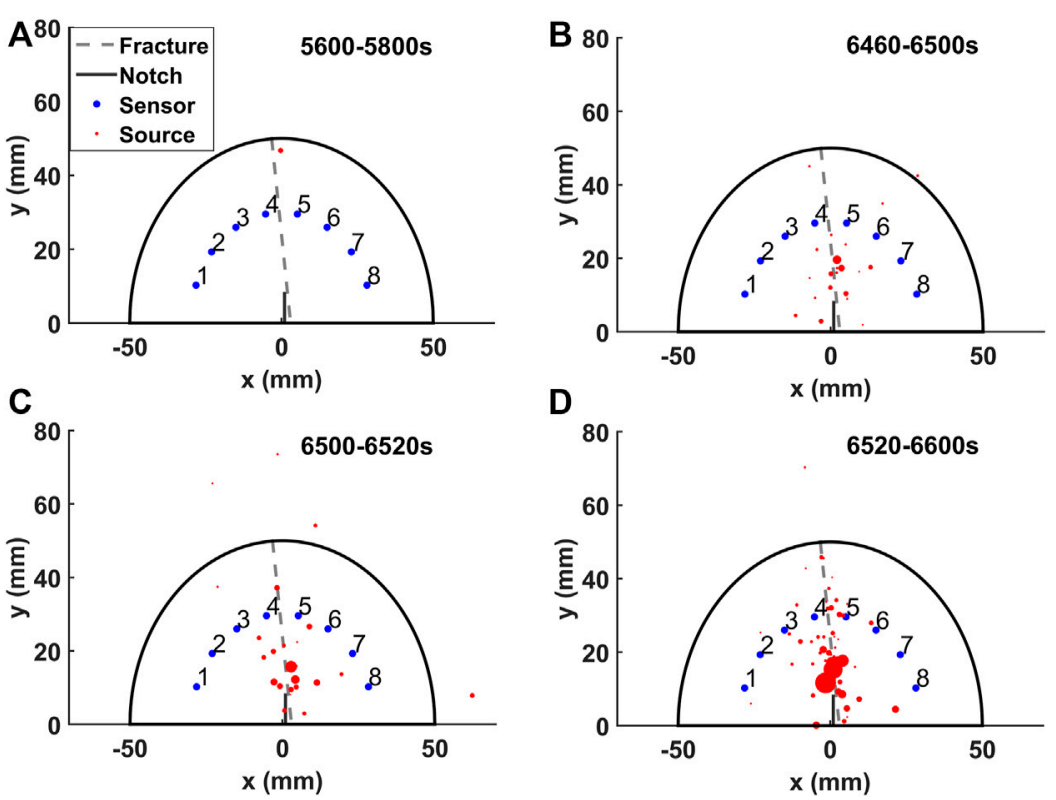

FIGURE 11 | Located AE events at different fracturing times (A) 5600-5800s, (B) 6460-6500s, (C) 6500-6520s, (D) 6520-6600s.

anisotropic method uses a stricter threshold $(\Delta t$ has a lower value) than the isotropic method since the latter's time threshold is calculated using a constant and relatively low velocity measured normal to the layer $\left(0^{\circ}\right.$ as shown in Figure 2), while the former's time threshold calculated using higher velocity depending on the direction.

\section{SOURCE LOCATION INCLUDING VELOCITY ANISOTROPY}

The source location can be determined by the source parameters inversion based on the known observational data. Source parameters, including coordinates and time of fracture initiation, can be estimated by resolving an inconsistent linear system (Geiger, 1912). As for an anisotropic medium, the function of the arrival time of the $k$ th sensor $f_{k}$ is expressed as

$$
f_{k}(\mathbf{m})=t+\frac{1}{V\left(\alpha_{k}\right)} \sqrt{\left(x_{k}-x\right)^{2}+\left(y_{k}-y\right)^{2}+\left(z_{k}-z\right)^{2}},
$$

where $\mathbf{m}=(t, x, y, z)^{T}$ is the vector of the source parameters in terms of the origin time $t$ and source coordinates $(x, y, z)$ to be inverted, $V\left(\alpha_{k}\right)$ is the calculated velocity based on ultrasonic measurements, and $\alpha_{k}$ is the angle of the $k$ th sensor to the $Z$ axis (Figure 8A), which can be expressed as

$$
\sin \alpha_{k}=\sqrt{\left(x_{k}-x\right)^{2}+\left(y_{k}-y\right)^{2}} / l \text { and } \cos \alpha_{k}=\left(z_{k}-z\right) / l,
$$

where $l=\sqrt{\left(x_{k}-x\right)^{2}+\left(y_{k}-y\right)^{2}+\left(z_{k}-z\right)^{2}}$. Given a certain point $\mathbf{m}_{0}=\left(t_{0}, x_{0}, y_{0}, z_{0}\right)^{T}, f_{k}(\mathbf{m})$ can be expanded using the first-order Taylor series as $f_{k}(\mathbf{m}) \approx f_{k}\left(\mathbf{m}_{0}\right)+\mathrm{A}\left(\mathbf{m}-\mathbf{m}_{0}\right)$
where $\mathrm{A}=\left[\begin{array}{cccc}\frac{\partial f_{1}}{\partial x} & \frac{\partial f_{1}}{\partial y} & \frac{\partial f_{1}}{\partial z} & \frac{\partial f_{1}}{\partial t} \\ \vdots & \vdots & \vdots & \vdots \\ \frac{\partial f_{k}}{\partial x} & \frac{\partial f_{k}}{\partial y} & \frac{\partial f_{k}}{\partial z} & \frac{\partial f_{k}}{\partial t}\end{array}\right]$. The source parameter update $\Delta \mathbf{m}=\mathbf{m}-\mathbf{m}_{0}$ can be recovered as

$$
\Delta \mathbf{m}=\left(\mathbf{A}^{\mathrm{T}} \mathbf{A}\right)^{-1} \mathbf{A}^{\mathrm{T}} \Delta f_{k},
$$

where $\Delta f_{k}=f_{k}(\mathbf{m})-f_{k}\left(\mathbf{m}_{0}\right)$, and $\mathrm{k}$ is greater than or equal to 4 . The vector of the source parameters is solved iteratively using the formula Eq. 11 until the specified error criterion is met

$$
\mathbf{m}_{n+1}=\mathbf{m}_{n}+\Delta \mathbf{m}_{n},
$$

where $n$ stands for the number of iteration.

This method is applied to experimental AE data. We compare the location results using isotropic velocity and anisotropic velocity, as shown in Figures 10A,B. These results are also calibrated with the actual fracture distribution from CT scanning as shown in Figures 10C,D, in which the amplitude of source events is displayed using varied size of dots. The valid location results must satisfy in the spatial domain $-50 \mathrm{~mm} \leq x \leq 50 \mathrm{~mm}, \quad 0 \leq y \leq 50 \mathrm{~mm}$ and $-25 \mathrm{~mm} \leq z \leq 25 \mathrm{~mm}$. Although 179 events are matched using the isotropic condition, only 40 sources are located using the isotropic location method, in which 31 sources are within the valid spatial domain (Figures 10A,C); while for 153 events matched 
using the anisotropic condition, 99 sources are located using the anisotropic location method, in which 71 sources are inside the valid spatial domain (Figures 10B,D). The number of located sources using the isotropic method is much less than that using the anisotropic method. In addition, the located sources using anisotropic velocity are better aligned with the fracture distribution, and the strong source events (displayed with larger dots) are more focused on the starting position of the fractures. To further demonstrate the result, we show four results using different processes (Figures 10C-F): isotropic matching and isotropic location (ISO + ISO), anisotropic matching and anisotropic location (ANI + ANI), isotropic matching and anisotropic location (ISO + ANI), and anisotropic matching and isotropic location (ANI + ISO). The located sources of anisotropic matching and anisotropic location show the best agreement with the CT scanning results in terms of fracture distribution and starting position. Therefore, it is very important to take into account the influence of anisotropy on the velocity for both AE event location and event-matching. Although there are several sources of weakamplitude located outside the semicircle sample (Figures 10B,D), this could be improved by more accurate arrival times selection and more receivers with wider geometry (receivers are placed only on planes $Z=-25 \mathrm{~mm}$ and $Z=25 \mathrm{~mm}$ in this study).

Finally, we analyze the located AE events at different stages of fracture, as shown in Figure 11. The first AE event is detected at 5800 s after loading (Figure 11A). After a quiet period of about 10 min, several acoustic emission events occurred during the 6460-6500s period (Figure 11B). These events appear around the fracture, but their distribution does not follow the strike of the fracture. In the period of 6500-6600s (Figures 11C,D), a large number of acoustic emission events are detected that developed along the fracture.

\section{CONCLUSION}

Event-matching is a necessary process for pinpointing a location. Traditional methods of event-matching and location can be affected by velocity anisotropy, leading to further unreliable source location results. In the event-matching process, if velocity anisotropy is ignored, many redundant events or false

\section{REFERENCES}

Akram, J., and Eaton, D. W. (2016). A Review and Appraisal of Arrival-Time Picking Methods for Downhole Microseismic Data. Geophysics 81 (2), KS67-KS87. doi:10.1190/geo2014-0500.1

Alkhalifah, T. (1998). Acoustic Approximations for Processing in Transversely Isotropic media. Geophysics 63 (2), 623-631. doi:10.1190/1. 1444361

Feng, X.-T., Young, R. P., Reyes-Montes, J. M., Aydan, Ö., Ishida, T., Liu, J.-P., et al. (2019). ISRM Suggested Method for In Situ Acoustic Emission Monitoring of the Fracturing Process in Rock Masses. Rock Mech. Rock Eng. 52 (5), 1395-1414. doi:10.1007/s00603-019-01774-z

Ge, M. C. (2013). Analysis of Source Location Algorithms Part II: Iterative Methods. J. Acoust. Emission 21, 29-51.

Geiger, L. (1912). Probability Method for the Determination of Earthquake Epicenters from the Arrival Time Only. Bull. St. Louis Univ. 8, 60-71.
AE events will be matched. Most of the source points detected by these false events are outside the sample, and the location results are incompatible with fracture distribution. In this article, we analyze the effect of anisotropy on the results of event-matching and location based on the three-point bend AE experiment. We achieve the event-matching and location taking into account the correction for anisotropic velocity, increasing the ratio of the number of detected sources to valid events, which may better help us in determining fracture characteristics. Real acoustic emission data applications show a clear improvement in location results over isotropic Geiger's results. The location results are also calibrated by CT scanning results, which show good consistency and confirm the improvement in fracture characteristics. In fact, both the velocity model and the sensor geometry have a large impact on the location results. If the sensors are distributed over a wide range of directions, we must not only take into account the anisotropy of the velocity model but also consider the anisotropy of velocity in eventmatching.

\section{DATA AVAILABILITY STATEMENT}

The raw data supporting the conclusion of this article will be made available by the authors, without undue reservation.

\section{AUTHOR CONTRIBUTIONS}

PW: acquisition and processing of AE data, data presentation, methodology, and original draft; FZ: the conception and design of the study, reviewing and editing, and supervision; X-YL: conceptualization, collected important background information, and manuscript editing.

\section{FUNDING}

This work was supported by the National Natural Science Foundation of China (U19B6003, 42122029) and the Strategic Cooperation Technology Projects of CNPC and CUPB (ZLZX2020-03).

Gibbons, S. J., and Ringdal, F. (2006). The Detection of Low Magnitude Seismic Events Using Array-Based Waveform Correlation. Geophys. J. Int. 165 (1), 149-166. doi:10.1111/j.1365-246x.2006.02865.x

Jin, S., and Stovas, A. (2020). S-wave in 2D Acoustic Transversely Isotropic media with a Tilted Symmetry axis. Geophys. Prospecting 68 (2), 483-500. doi:10.1111/ 1365-2478.12856

Jin, S., and Stovas, A. (2018). S-wave Kinematics in Acoustic Transversely Isotropic media with a Vertical Symmetry axis. Geophys. Prospecting 66 (6), 1123-1137. doi:10.1111/1365-2478.12635

King, A., and Talebi, S. (2007). Anisotropy Effects on Microseismic Event Location. Pure Appl. Geophys. 164 (11), 2141-2156. doi:10.1007/s00024-007-0266-8

Li, J. H., and Qi, G. (2009). Improving Source Location Accuracy of Acoustic Emission in Complicated Structures. Journal of Nondestructive Evaluation 28, 1-8. doi:10.1007/s10921-009-0042-Z

Liu, Z., Zhang, F., and Li, X. (2019). Elastic Anisotropy and its Influencing Factors in Organic-Rich marine Shale of Southern China. Sci. China Earth Sci. 62, 1805-1818. doi:10.1007/s11430-019-9449-7 
Lonardelli, I., Wenk, H.-R., and Ren, Y. (2007). Preferred Orientation and Elastic Anisotropy in Shales. Geophysics 72, D33-D40. doi:10.1190/1. 2435966

López Comino, J. A., Heimann, S., Cesca, S., Milkereit, C., Dahm, T., and Zang, A. (2017). Automated Full Waveform Detection and Location Algorithm of Acoustic Emissions from Hydraulic Fracturing Experiment. Procedia Engineering 191, 697-702. doi:10.1016/j.proeng.2017.05.234

Maeda, N. (1985). A Method for Reading and Checking Phase Time in AutoProcessing System of Seismic Wave Data. Jssj 38, 365-379. doi:10.4294/ zisin1948.38.3_365

Mavko, G., Mukerji, T., and Dvorkin, J. (2003). The Rock Physics Handbook-Tools for Seismic in Porous media. Cambridge University Press.

Maxwell, S. C., Bennett, L., and Jones, M. (2010). Anisotropic Velocity Modeling for Microseismic Processing: Part 1-Impact of Velocity Model Uncertainty: SEG Technical Program Expanded Abstracts 2010. Denver,Colorado: Soc. Expl. Geophys., 2130-2134.

Song, F., Kuleli, H. S., Toksöz, M. N., Ay, E., and Zhang, H. (2010). An Improved Method for Hydrofracture-Induced Microseismic Event Detection and Phase Picking. Geophysics 75 (6), A47-A52. doi:10.1190/1.3484716

Thomsen, L. (1986). Weak Elastic Anisotropy. Geophysics 51 (10), 1954-1966. doi:10.1190/1.1442051

Van Dok, R., Fuller, B., Engelbrecht, L., Sterling, M., and Hipoint, A. (2011). Seismic Anisotropy in Microseismic Event Location Analysis. The Leading Edge 30 (7), 766-770. doi:10.1190/1.3609091

Vernik, L., and Nur, A. (1992). Ultrasonic Velocity and Anisotropy of Hydrocarbon Source Rocks. Geophysics 57 (5), 727-735. doi:10.1190/1. 1443286

Wang, Z. (2002). Seismic Anisotropy in Sedimentary Rocks, Part 1: A Single-plug Laboratory Method. Geophysics 67 (5), 1415-1422. doi:10.1190/1.1512787

Wuestefeld, A., Greve, S. M., Näsholm, S. P., and Oye, V. (2018). Benchmarking Earthquake Location Algorithms: A Synthetic Comparison. Geophysics 83 (4), KS35-KS47. doi:10.1190/geo2017-0317.1
Zhang, F. (2019). A Modified Rock Physics Model of Overmature Organic-Rich Shale: Application to Anisotropy Parameter Prediction from Well Logs. J. Geophys. Eng. 16 (1), 92-104. doi:10.1093/jge/gxy008

Zhang, F. (2017). Estimation of Anisotropy Parameters for Shales Based on an Improved Rock Physics Model, Part 2: Case Study. J. Geophys. Eng. 14, 238-254. doi:10.1088/1742-2140/aa5afa

Zhang, F., Li, X.-y., and Qian, K. (2017). Estimation of Anisotropy Parameters for Shale Based on an Improved Rock Physics Model, Part 1: Theory. J. Geophys. Eng. 14, 143-158. doi:10.1088/1742-2140/14/1/143

Zhang, H. L., Zhu, G. M., and Wang, Y. H. (2013). Automatic Microseismic Event Detection and Picking Method. Geophys. Geochemical Exploration 37 (2), 269-273.

Zhou, Z. L., Zhou, J., Dong, L. J., Cai, X., Rui, Y. C., and Ke, C. T. (2017). Experimental Study on the Location of an Acoustic Emission Source Considering Refraction in Different media. Sci. Rep. 7 (1), 7472. doi:10. 1038/s41598-017-07371-w

Conflict of Interest: The authors declare that the research was conducted in the absence of any commercial or financial relationships that could be construed as a potential conflict of interest.

Publisher's Note: All claims expressed in this article are solely those of the authors and do not necessarily represent those of their affiliated organizations, or those of the publisher, the editors, and the reviewers. Any product that may be evaluated in this article, or claim that may be made by its manufacturer, is not guaranteed or endorsed by the publisher.

Copyright (c) 2022 Wang, Zhang and Li. This is an open-access article distributed under the terms of the Creative Commons Attribution License (CC BY). The use, distribution or reproduction in other forums is permitted, provided the original author(s) and the copyright owner(s) are credited and that the original publication in this journal is cited, in accordance with accepted academic practice. No use, distribution or reproduction is permitted which does not comply with these terms. 